\title{
Asymptotic behavior of nonoscillatory solutions of second order functional differential equations
}

\section{Takaŝi Kusano and Hiroshi Onose}

The asymptotic behavior of nonoscillatory solutions of the second order functional differential equation

$$
\left(r(t) y^{\prime}(t)\right)^{\prime}+a(t) f(y(g(t)))=b(t)
$$

is studied. First, in the case when $a(t)$ is oscillatory, sufficient conditions are given in order that all bounded nonoscillatory solutions of (*) approach zero as $t \rightarrow \infty$. Secondly, in the case when $a(t)$ is nonnegative, conditions are provided under which all nonoscillatory solutions of $(*)$ tend to zero as $t \rightarrow \infty$.

\section{Introduction}

We consider the differential equation

$$
\left(r(t) y^{\prime}(t)\right)^{\prime}+a(t) f(y(g(t)))=b(t),
$$

where $a(t), b(t), g(t), r(t)$ are continuous on $[\tau, \infty)$, and $f(y)$ is continuous on $(-\infty, \infty)$. The following conditions are assumed to hold throughout the paper:

Received 8 July 1975. 
(a) $\lim _{t \rightarrow \infty} g(t)=\infty$;

(2) (b) $r(t)>0$ and $\lim _{t \rightarrow \infty} R(t)=\infty$, where $R(t)=\int_{\tau}^{t} \frac{d s}{r(s)}$;

(c) $y f(y)>0$ for $y \neq 0, \underset{y \rightarrow \infty}{\liminf } f(y)>0$ and $\lim \sup f(y)<0$. $y \rightarrow-\infty$

We restrict our consideration to those solutions of (1) which exist on some half-line $[T, \infty)$, where $T$ may depend on the particular solution, and are nontrivial in any neighborhood of infinity. Such a solution is said to be nonoscillatory if it is eventually positive or eventually negative. Otherwise the solution is said to be oscillatory.

The problem is to provide sufficient conditions which ensure that all (bounded) nonoscillatory solutions of (1) tend to zero as $t \rightarrow \infty$. Since the work of Hammett [2] this problem has received a considerable amount of attention and a number of results have been obtained; see, for example, Grimmer [1], Londen [3], Singh [4], [5], and Singh and Dahiya [6]. The purpose of this paper is to add new results to this problem. First, we consider the case where $a(t)$ is oscillatory and give conditions under which all bounded nonoscillatory solutions of (1) tend to zero as $t \rightarrow \infty$. Secondly, we examine the case where $a(t)$ is nonnegative and provide sufficient conditions in order that all nonoscillatory solutions of ( 1 ) approach zero as $t \rightarrow \infty$.

\section{The case where $a(t)$ is oscillatory}

We need the following lemma which is a generalization of a lenma of Staikos and Sficas [7].

LEMMA. Consider the differential equation

$$
u^{\prime}-\frac{\rho^{\prime}(t)}{\rho(t)} u+\frac{\rho^{\prime}(t)}{\rho(t)} \phi(t)=0,
$$

where $\phi(t)$ is continuous on $[T, \infty), \rho(t)$ is continwously differentiable on $[T, \infty)$, and

$$
\rho(t)>0, \quad \rho^{\prime}(t)>0, \lim _{t \rightarrow \infty} \rho(t)=\infty .
$$


Let $u(t)$ be the solution of $(3)$ on $[T, \infty)$ satisfying $u(T)=0$.

If $\lim _{t \rightarrow \infty}|\phi(t)|=\phi^{*}$ exists in the extended real line $R^{\#}$, then $\lim _{t \rightarrow \infty}|u(t)|=u^{*}$ exists in $R^{\#}$. In particular, $\phi^{*}=\infty$ implies $u^{*}=\infty$.

Proof. The solution $u(t)$ is given explicitly by the formula

$$
u(t)=-\rho(t) \int_{T}^{t} \frac{\rho^{\prime}(s)}{\rho^{2}(s)} \phi(s) d s, \quad t \geq T .
$$

The existence of $\phi^{*}$ in $R^{\#}$ implies that the improper integral

$$
\lim _{t \rightarrow \infty} \int_{T}^{t} \frac{\rho^{\prime}(s)}{\rho^{2}(s)} \phi(s) d s=\Phi^{*}
$$

exists in $R^{\#}$. If $\Phi^{*} \neq 0$, then clearly $\lim _{t \rightarrow \infty}|u(t)|=\infty$. If $\Phi^{*}=0$, then by I'Hospital's Rule,

$$
\lim _{t \rightarrow \infty}|u(t)|=\lim _{t \rightarrow \infty}\left|\left(-\int_{T}^{t} \frac{\rho^{\prime}(s)}{\rho^{2}(s)} \phi(s) d s\right)^{\prime} /\left(\frac{I}{\rho(t)}\right)^{\prime}\right|=\phi^{*} .
$$

In what follows we use the notation $a^{+}(t)=\max \{a(t), 0\}$, $a^{-}(t)=\max \{-a(t), 0\}$.

THEOREM 1. Every bounded nonoscizlatory solution of (I) tends to zero as $t \rightarrow \infty$ if the following assumptions are satisfied:

$$
\begin{aligned}
& \int^{\infty} R(t) a^{+}(t) d t=\infty, \\
& \int^{\infty} R(t) a^{-}(t) d t<\infty, \\
& \int^{\infty} R(t)|b(t)| d t<\infty .
\end{aligned}
$$

Proof. Let $y(t)$ be a bounded nonoscillatory solution of (1). Suppose that $y(t)>0$ for $t \geq t_{0}$. The case $y(t)<0$ can be handled similarly. By (2) (a) there exists $t_{1} \geq t_{0}$ such that $g(t) \geq t_{0}$ for $t \geq t_{1}$. Thus, $y(g(t))>0$ for $t \geq t_{1}$. Multiplying (1) by $R(t)$ and 
integrating from $t_{1}$ to $t$, we obtain

(7) $\begin{aligned} \int_{t_{1}}^{t} R(s)\left(r(s) y^{\prime}(s)\right)^{\prime} d s & +\int_{t_{1}}^{t} R(s) a^{+}(s) f(y(g(s))) d s \\ & =\int_{t_{1}}^{t} R(s) b(s) d s+\int_{t_{1}}^{t} R(s) a^{-}(s) f(y(g(s))) d s .\end{aligned}$

An integration by parts yields

$$
\int_{t_{I}}^{t} R(s)\left(r(s) y^{\prime}(s)\right)^{\prime} d s=R(t) r(t) y^{\prime}(t)-R\left(t_{I}\right) r\left(t_{I}\right) y^{\prime}\left(t_{1}\right)-\int_{t_{I}}^{t} y^{\prime}(s) d s \text {. }
$$

By a mere reinterpretation this says that the function $u(t)=\int_{t_{1}}^{t} y^{\prime}(s) d s$ satisfies the differential equation

$$
u^{\prime}-\frac{R^{\prime}(t)}{R(t)} u+\frac{R^{\prime}(t)}{R(t)} \Phi(t)=0,
$$

where

$$
\Phi(t)=-\int_{t_{1}}^{t} R(s)\left(r(s) y^{\prime}(s)\right)^{\prime} d s-R\left(t_{1}\right) r\left(t_{1}\right) y^{\prime}\left(t_{1}\right)
$$

Since $u\left(t_{1}\right)=0$ and since $R(t)>0, R^{\prime}(t)>0, \lim _{t \rightarrow \infty} R(t)=\infty$ by

(2) (b), we can apply the above lemma to (8) to conclude that $\lim _{t \rightarrow \infty}|u(t)|$ exists in $R^{\#}$ whenever $\lim _{t \rightarrow \infty}|\Phi(t)|$ exists in $R^{\#}$.

We examine the following two possible cases:

$$
\begin{aligned}
& \int_{t_{1}}^{\infty} R(t) a^{+}(t) f(y(g(t))) d t=\infty, \\
& \int_{t_{1}}^{\infty} R(t) a^{+}(t) f(y(g(t))) d t<\infty .
\end{aligned}
$$

Suppose (10) holds. Since the right-hand side of (7) remains bounded as $t \rightarrow \infty$ on account of $(5),(6)$, and the boundedness of $y(t)$, it follows 
from (7) and (10) that

$$
\lim _{t \rightarrow \infty} \int_{t_{1}}^{t} R(s)\left(r(s) y^{\prime}(s)\right) \cdot d s=-\infty
$$

which implies that the function $\Phi(t)$ defined by (9) tends to $\infty$ as $t+\infty$. Hence, by the lemma, $\lim _{t \rightarrow \infty}|u(t)|=\infty$, and consequently

$\lim y(t)=\infty$. This, however, contradicts the boundedness of $y(t)$ and $t \rightarrow \infty$

thus (10) is impossible.

Now it follows from (7) and (11) that the limit on the left-hand side of (12) exists as a finite number; that is, $\lim \Phi(t)$ is finite. Applying $t \rightarrow \infty$

the lemma again, we see that $\lim _{t \rightarrow \infty}|u(t)|$ exists in $R^{\#}$. Obviously this limit must be finite. Therefore, $y(t)$ must tend to a finite limit as $t \rightarrow \infty$. On the other hand, using (2) (c), (4) and (1I), we have

$$
\lim _{t \rightarrow \infty} \inf y(g(t))=\underset{t \rightarrow \infty}{\lim \inf } y(t)=0 \text {. }
$$

Consequently we conclude that $\lim _{t \rightarrow \infty} y(t)=0$, completing the proof.

Retracing the above proof it is easy to see that the conclusion of Theorem $I$ still holds if the roles of $a^{+}(t)$ and $a^{-}(t)$ are interchanged. Accordingly we have the following theorem.

THEOREM 2. Every bounded nonoscizlatory solution of (1) tends to zero as $t \rightarrow \infty$ if the following conditions are satisfied:

$$
\begin{aligned}
& \int^{\infty} R(t) a^{+}(t) d t<\infty, \\
& \int^{\infty} R(t) a^{-}(t) d t=\infty, \\
& \int^{\infty} R(t)|b(t)| d t<\infty .
\end{aligned}
$$

COROLLARY 1. Every bounded nonoscizlatory solution of the equation

$$
y^{\prime \prime}(t)+a(t) f(y(g(t)))=b(t)
$$


tends to zero as $t \rightarrow \infty$ if either

$$
\int^{\infty} t a^{+}(t) d t=\infty, \int^{\infty} t a^{-}(t) d t<\infty, \quad \int^{\infty} t|b(t)| d t<\infty, .
$$

or

$$
\int^{\infty} t \alpha^{+}(t) d t<\infty, \quad \int^{\infty} t a^{-}(t) d t=\infty, \quad \int^{\infty} t|b(t)| d t<\infty
$$

EXAMPLES (1) Consider the differential equation

$$
\left(t^{-1} y^{\prime}(t)\right)^{\prime}+t^{-2} y^{3}(\gamma t)=3 t^{-4}+\gamma^{-3} t^{-5}
$$

for $t \geq 1$, where $\gamma$ is a positive constant (possibly greater than 1 ). Here $f(y)=y^{3}, g(t)=\gamma t$,

$$
r(t)=t^{-1}, a^{+}(t)=t^{-2}, a^{-}(t)=0 \text {, and } b(t)=3 t^{-4}+\gamma^{-3} t^{-5} \text {. }
$$

We can take $R(t)=t^{2}$ and see that all conditions of Theorem 1 are satisfied. Hence all bounded nonoscillatory solutions of (13) approach zero as $t \rightarrow \infty$. In fact, $y(t)=t^{-I}$ is a bounded nonoscillatory solution of (13).

(2) Consider the equation

$$
\left(t y^{\prime}(t)\right)^{\prime}-t^{-1}(\log t)^{-1} y(\log t)=\left(1-3 t+t^{2}\right) e^{-t}-t^{-2}
$$

for $t \geq 1$, which has $y(t)=t e^{-t}$ as a bounded nonoscillatory solution. Here $f(y)=y, g(t)=\log t, \quad x(t)=t$,

$$
a^{+}(t)=0, a^{-}(t)=t^{-1}(\log t)^{-1} \text {, and } b(t)=\left(1-3 t+t^{2}\right) e^{-t}-t^{-2} .
$$

All the conditions of Theorem 2 are satisfied with $R(t)=10 g t$. It follows that all bounded nonoscillatory solutions of (14) tend to zero as $t \rightarrow \infty$.

\section{The case where $a(t)$ is nonnegative}

In this section we examine the equation ( 1 ) in which $a(t)$ is nonnegative and provide conditions under which all nonoscillatory solutions are necessarily bounded and tend to zero as $t \rightarrow \infty$.

THEOREM 3. Assume that $a(t) \geq 0$ and 


$$
\begin{gathered}
\int^{\infty} a(t) d t=\infty, \\
\int^{\infty} R(t)|b(t)| d t<\infty .
\end{gathered}
$$

Then all nonoscizlatory solutions of (1) tend to zero as $t \rightarrow \infty$.

Proof. Let $y(t)$ be a nonoscillatory solution of (1) such that $y(g(t))>0$ for $t \geq t_{1}$. A parallel argument holds if $y(g(t))<0$ for $t \geq t_{1}$. From (I) we obtain

(17) $\quad r(t) y^{\prime}(t)-r\left(t_{1}\right) y^{\prime}\left(t_{1}^{\prime}\right)+\int_{t_{1}}^{t} a(s) f(y(g(s))) d s=\int_{t_{1}}^{t} b(s) d s$,

(18) $\int_{t_{1}}^{t} R(s)\left(r(s) y^{\prime}(s)\right)^{\prime} d s+\int_{t_{1}}^{t} R(s) a(s) f(y(g(s))) d s=\int_{t_{1}}^{t} R(s) b(s) d s$.

Suppose that

$$
\int_{t_{1}}^{\infty} R(t) a(t) f(y(g(t))) d t=\infty
$$

Then, letting $t \rightarrow \infty$ and using (16), we see that the first integral in (18) tends to $-\infty$ as $t \rightarrow \infty$, so that $\lim _{t \rightarrow \infty} \Phi(t)=\infty$, where $\Phi(t)$ is defined by (9). From the lemma applied to (8) it follows that $u(t)=\int_{t_{1}}^{t} y^{\prime}(s) d s$ tends to $\infty$ as $t \rightarrow \infty$. Consequently, we have $\lim _{t \rightarrow \infty} y(t)=\infty$. Combining this with (2) (c) and (15), we find (20)

$$
\int_{t_{1}}^{\infty} a(t) f(y(g(t))) d t=\infty
$$

Since (16) implies that the right-hand side of (17) is bounded, we have from $(17)$ and (20),

$$
\lim _{t \rightarrow \infty} r(t) y^{\prime}(t)=-\infty
$$


from which we conclude with the use of (2) (b) that $\lim _{t \rightarrow \infty} y(t)=-\infty$. But this contradicts the fact that $y(t)$ is positive. Hence (19) is impossible and we must have

$$
\int_{t_{1}}^{\infty} R(t) \alpha(t) f(y(g(t))) d t<\infty .
$$

Letting $t \rightarrow \infty$ again in (18), we see that $\lim _{t \rightarrow \infty} \Phi(t)$ exists and is finite. According to the lemma it follows that $\lim _{t \rightarrow \infty} u(t)$ exists in $R^{\#}$. This limit must be finite, since otherwise we would be led to a contradiction to the positivity of $y(t)$ as before. Therefore, there exists a finite limit $\lim y(t)$. On the other hand, (2l) implies $t_{t \rightarrow \infty}$

$$
\int_{t_{1}}^{\infty} a(t) f(y(g(t))) d t<\infty,
$$

and from (2) (c), (15), and (22) it is easily verified that

$$
\lim _{t \rightarrow \infty} \inf y(g(t))=\underset{t \rightarrow \infty}{\lim \inf } y(t)=0 \text {. }
$$

Thus it follows that $\lim _{t \rightarrow \infty} y(t)=0$, and the proof is complete.

COROLLARY 2. AZZ nonoscizzatory solutions of the equation

$$
y^{\prime \prime}(t)+a(t) f(y(g(t)))=b(t)
$$

approach zero as $t \rightarrow \infty$ if $a(t) \geq 0$,

$$
\int^{\infty} a(t) d t=\infty \text { and } \int^{\infty} t|b(t)| d t<\infty .
$$

EXAMPLES (3). Consider the equation

$$
\left(t^{-1} y^{\prime}(t)\right)^{\prime}+t^{-1} y^{3}(\gamma t)=\left(3+\gamma^{-3}\right) t^{-4}
$$

for $t \geq 1$, where $\gamma$ is a positive constant. This equation possesses a nonoscillatory solution $y(t)=t^{-1}$ tending to zero as $t \rightarrow \infty$. Since all conditions of Theorem 3 are satisfied, all other nonoscillatory solutions of (23) also approach zero as $t \rightarrow \infty$. 
(4) Consider the equation

$$
\left(t y^{\prime}(t)\right)^{\prime}+t^{-1}(\log t)^{-1} y(\log t)=\left(1-3 t+t^{2}\right) e^{-t}+t^{-2}
$$

for $t \geq 1$. Clearly, this equation satisfies the assumptions of Theorem 3. Therefore, all of its nonoscillatory solutions tend to zero as $t \rightarrow \infty$. In fact, $y(t)=t e^{-t}$ is a nonoscillatory solution of (24) having this property.

\section{References}

[1] R. Grimmer, "On nonoscillatory solutions of a nonlinear differential equation", Proc. Amer. Math. Soc. 34 (1972), 118-120.

[2] Michael E. Hammett, "Nonoscillation properties of a nonlinear differential equation", Proc. Amer. Math. Soc. 30 (1971), 92-96.

[3] Stig-Olof Londen, "Some nonoscillation theorems for a second order nonlinear differential equation", SIAM J. Math. Anal. 4 (1973), $460-465$.

[4] Bhagat Singh, "Nonoscillation of forced fourth order retarded equations", SIAM J. AppZ. Math. 28 (1975), 265-269.

[5] Bhagat Singh, "Asymptotic nature of nonoscillatory solutions of $n$th order retarded differential equations", SIAM J. Math. AnaZ. (to appear).

[6] Bhagat Singh and R.S. Dahiya, "On oscillation of second-order retarded equations", J. Math. Anal. Appl. 47 (1974), 504-512.

[7] V.A. Staikos and Y.G. Sficas, "Forced oscillations for differential equations of arbitrary order", J. Differential Equations 17 (1975), 1-11.

Department of Mathematics,

Faculty of Science,

Hiroshima University,

Hiroshima,

Japan;
Department of Mathematics, Faculty of General Education, Ibaraki University, Mito, Japan. 